\title{
Article
}

\section{Glutamic acid promotes hair growth in mice}

Carlos Poblete Jara ${ }^{1,3}$, Beatriz de Andrade Berti ${ }^{1,3}$, Natalia Ferreira Mendes ${ }^{1,3}$, Daiane F. Engel 2,3 , Ariane Maria Zanesco ${ }^{2,3}$, Gabriela Freitas Souza ${ }^{2,3}$, Lício Augusto Velloso ${ }^{2,3}$, Eliana Pereira de Araujo ${ }^{1,3}$

${ }^{1}$ Faculty of Nursing, ${ }^{2}$ Faculty of Medical Sciences, ${ }^{3}$ Laboratory of Cell Signaling, Obesity and Comorbidities Research Center, University of Campinas, Brazil

Carlos P. Jara (ORCID: 0000-0001-5444-721X)

Natália F. Mendes (ORCID: 0000-0001-9032-1411)

Ariane Maria Zanesco (ORCID: 0000-0003-4842-1421)

Daiane Fátima Engel (ORCID:0000-0002-1950-196X)

Eliana P. Araújo (ORCID: 0000-0002-7539-8477)

Corresponding author:

Eliana Pereira Araújo, Ph.D., Associate Professor

Faculty of Nursing, University of Campinas, UNICAMP

Tessalia Vieira de Camargo St, 126 - 13083-887 Campinas - SP - Brazil

E-mail: earaujo@unicamp.br; pa.eliana@gmail.com

Financial support: Coordination of Improvement of Higher-Level Personnel of Brazil (CAPES)

Short running title: Glutamic acid and skin

Keywords: Glutamic acid; skin; hair growth; hair follicle. 


\section{Abstract}

Glutamic Acid is the main excitatory neurotransmitter in neurons. Abnormal distributions of the glutamic acid receptors have been shown in hyper proliferative models such as psoriasis and skin regeneration. However, the biological function of glutamic acid in the skin remains unclear. Using ex vivo, in vivo and in silico approaches, we showed for the first time that exogenous glutamic acid promotes hair growth and keratinocyte proliferation. Topical application of glutamic acid decreased expression of genes related to apoptosis signaling in the skin. Also, we showed Glutamic acid increased viability and proliferation in cultured human keratinocyte. For the first time, we identified the excitotoxic GA concentration and we provided evidence for the existence of a novel skin signaling pathway mediated by a neurotransmitter controlling keratinocyte and hair follicle proliferation. In perspective, we anticipate our results could be the starting point to elucidate how exogenous glutamic acid from food intake or even endogenous GA from neuropsychiatric disorders modulate skin diseases.

Keywords: Glutamic acid; skin; hair growth; hair follicle. 


\section{Introduction}

Glutamic acid $(G A)$ is the major excitatory neurotransmitter in the mammalian central nervous system and it is predominantly associated with excitatory synaptic neurotransmission (1). Previous reports have identified skin GA receptors and transporters across different species. Skin GA receptors subunits (Grin1, Grin2a, Gria2, and Grm1), and transporters (SIc1a1 and SIc1a2) were identified in rat skin (2), mouse skin (Grin1) and human keratinocyte (GRIN1) (3, 4)(5). Moreover, epidermis, hair follicles and sebaceous glands showed glutamate positive immunofluorescence (6).

Physiologically, the glutamatergic signaling through NMDARs in hair follicle cells was previously shown. Glutamic acid signaling is essential for innervation and differentiation of Grin1 positive Schwann cells during piloneural collar development in hair follicles (4). Specifically, NMDA receptors are highly expressed in type I and type II terminal Schwann cells. These cells are circumferential localized in the bulge border and covering a majority of outer root sheath keratinocytes in the isthmus (From Excitatory glutamate is essential for development and maintenance of the piloneural mechanoreceptor).

Previous reports have shown the effects of topical application of GA in wounded skins. In vivo studies showed that $1 \%$ L-Glutamic acid loaded hydrogels speeded up wound closure by increasing collagen synthesis and crosslinking in diabetic rats (7). Also, 1\% L-Glutamic acid loaded hydrogels accelerated vascularization and macrophage recruitment into the diabetic wound (7). Moreover, topical D-Glutamic acid has also improved damaged skin by accelerating the barrier recovery (3), altogether suggesting a positive effect in skin repair.

We found five patent documentation using topical GA and derived molecules for hair growth stimulation, however, no scientific reports describing how GA could stimulate hair growth or epidermal cell proliferation. In this way, the biological effect of topical GA application in healthy skin is still unclear. Here, we evaluated the effect of topical GA in the back of healthy mice. We hypothesized whether GA could affect proliferation and viability in skin cells. In this manuscript, we have reported the preparation of L-glutamic acid formulations and its potential in hair stimulation. 


\section{RESULTS}

\section{Glutamic acid increases human keratinocytes viability and proliferation}

We hypothesized whether GA could stimulate proliferation and survival, keratinocytes will grow even in a confluence context (Fig. 1a). To do that, we removed any traces of FBS from the medium formulas due to the growth factors present in the bovine serum. By the end of 2 days of GA exposure, and even after $100 \%$ confluence, keratinocyte increased viability and proliferation. Indeed, we identified keratinocytes follow a Gaussian distribution pattern of viability after GA exposure (Fig. 1c). After 1 day of treatment, $100 \mu \mathrm{M}$ and 10mM GA concentrations increased human keratinocyte viability (Fig. 1b). These differences were higher after 2 days of $\mathrm{GA}$ exposure (Fig. 1c). The $100 \mu \mathrm{M}, 1 \mathrm{mM}$, and $10 \mathrm{mM}$ GA concentrations increased keratinocyte viability within 2 days of treatment (Fig. 1c). As 10mM and 100mM GA concentrations showed opposite effects in the viability test (proliferative and excitotoxic respectively), we evaluated whether proliferation could be affected after 2 days of GA exposure (Fig. 1d). Consistently with viability results, BrdU positive keratinocytes were higher in the $10 \mathrm{mM}$ group (Fig. 1g). On the other hand, we identified an excitotoxic concentration at $100 \mathrm{mM}$ GA. Keratinocytes treated with GA decreased cell viability after 1 day (Fig. 1b) and 4 days of treatment (Supplementary 1a).

\section{Topical Glutamic acid decreases apoptotic related genes}

To understand how GA promotes proliferation and improves viability, we evaluated apoptotic related gene expression. After 14 days of topical GA treatment, $0.1 \%, 0.5 \%$ and $10 \%$ decreased Blc2 gene expression (Fig 1h). Also, 10\% GA treatment decreased BAX expression (Fig 1h). However, we found no differences in Casp9 expression (Fig. 1h). Next, we evaluate whether topical GA could stimulate expression of genes related to inflammatory response. We found no differences in II1- $\beta$, Tnf- $\alpha$, nor II10. However, F4/80, a well-known marker of macrophage populations, and Monocyte Chemoattractant Protein-1 (Mcp1) gene expression were increased after 14 days of $1 \%$ GA (Fig. 1h). Additionally, topical GA $10 \%$ decreased the expression of Glutamate Ionotropic Receptor NMDA Type Subunit 1 (Grin1) with no differences in Glutamate Aspartate Transporter 1 (Glast) expression (Fig 1h).

\section{Topical Glutamic acid accelerates hair growth in healthy mice}

To identify if our in vitro results could also be found in in vivo keratinocytes, we applied 4 different concentrations of GA on the back of Swiss mice (Fig. 1e, 2a). Surprisingly, 1\% and 10\% 
of GA speeded up hair growth after 14 days of topical treatment (Fig. 2a). Also, photomicrograph showed that GA increased external root sheath across all GA concentration (Fig. 2b, supplementary 1b) with no hyperkeratosis effect. Consistently, we also identified increased BrdU positive cells in the hair follicles and epidermal layer after 14-days GA topical treatment (Fig. 3a, d).

\section{Exogenous topical glutamic acid increased vascularization}

We identified macroscopic differences in vascularization after 14 days treatment. The $0.5 \%$ and 10\% GA topical application increased skin vascularization (Fig. 2c-d). Following these results, we evaluated whether GA could induce expression of vascular gene regulators. We found that 1\% GA topical treatment increased the Hypoxia Inducible Factor 1 Subunit Alpha (Hif1a), a master regulator of vascularization (8,9) (Fig. 2e). Also, 1\% GA topical treatment increased the Vascular Endothelial Growth Factor A (Vegf), which induces proliferation and migration of vascular endothelial cells and it is essential for physiological angiogenesis (1012)(Fig. 2e).

\section{Single cell RNA sequencing analysis showed differences in Glutamate receptor and transporter localization between mice and human}

We evaluated glutamate receptor expression, using immunostaining, quantitative PCR, and single-cell RNA sequencing techniques. We identified that subunits Grin1, Grin2a, Grin2b, and Grin2c of NMDA receptor are expressed in the skin (Fig. 3b-c) and Grin2b is expressed specifically in keratin $14+$ cells (Fig. $3 b-c$ ). To improve the accuracy, and due to the wide number of subunits (5 GA receptor families with 26 subunits) we used an RNA single cell approach (Fig. 4a). Using public transcriptome libraries of skin tissue, we analyzed $\sim 73,000$ mice and human epidermal cells from back (mice), foreskin, trunk, and scalp (human). This cross-species analysis showed a similar percentage (5\%) of "glutamatergic" epidermal subpopulation in the skin (Fig. 6b-c). In humans, we identified NMDA receptors as the highest expressed subunits in basal layer and hair follicular cell clusters, specifically GRIN2A subunit (Fig. 4b). Also, we identify that melanocytes expressed Glutamate lonotropic Receptor Delta Type Subunit 1 (GRD1) (Fig. 4b). Additionally, we identified Excitatory Amino Acid Transporter 4 (SLC1A6) expression in granular cells, and Excitatory Amino Acid Transporter 1 (SLC1A3) expression in basal layer cells (Fig. 4c). In mice, we identified that Grin2d (in Sebaceous Gland) and Grik1 (in the hair follicle bulge) are the highest expressed subunits (Fig. 4d). Additionally, 
we identified the expression of Excitatory Amino Acid Transporter 3 (SIc1a3) (Fig. 4e) and Slc1a1 in $50 \%$ of all Sebaceous Gland cells (Fig. 4e)

To predict a glutamic acid-mediated cell signaling pathway between GA signal and hair follicle related genes, we used computational interaction network analysis STRING. In this way, we used glutamate receptor pathway genes and hair cycle genes ontologies (Supplementary 2). We found that GA receptors interact with Hair cycle genes through the Tyrosine-protein kinase Fyn, Ca 2+ /calmodulin-dependent protein kinase II (CaMKII), and Protein kinase B (Akt) (Fig. 4f). Additionally, we found $B C / 2$ as a common apoptotic regulator between both hair cycle and GA pathway (Fig. 4f). To confirm that, we evaluated the protein expression of Fyn, CaMKII and Akt in the 14-day topical GA treated mice (Fig. 4g-i). We found no differences in Fyn quantification (Fig. 4i). However, we confirmed that AKT2 phosphorylation increased after 14 days of topical 1\% GA treatment (Fig. 4g). Also, pCaMKII increased after 14 days of topical $10 \%$ GA treatment (Fig. 4h). 


\section{Discussion}

Interestingly, we found no scientific reports describing GA treatment or even GA effect in hair growth or epidermal cell proliferation. However, we found five patent documentation using topical GA (or derived molecules) for hair growth. One of these patents described the use of GA as a hair conditioner (patent number CN106580722A, China) for "hair restoration" and alopecia prevention. Other patent documentation showed a Poly-Gamma-GA composition for preventing hair loss and promoting hair growth (KR20150110149A, Korea). Also, synthetic compounds of GA attached to minoxidil for keratinocyte growth and hair growth in humans (USO05801150A, USA). Furthermore, a possible 2 to 12\% GA topical cream could be used for combating hair loss or alopecia in humans (FR2939038B1, France). Additionally, 42 different molecules derived from L-Glutamic Acid were described as hair growth promoters (PI9302024A, Brazil). However, these patent documentations do not show any cell signaling to explain mechanisms of hair growth stimulation after GA application. In this way, the clinical importance of skin glutamic acid signaling is highly desirable.

Here, we tried to understand more about GA functions in the skin. First, using an in vitro approach we challenged 100\% confluence human keratinocytes (without FBS or growth factor supply) to continue growing. Our result showed that GA increases proliferation and viability in keratinocytes. In this way, GA could represent a remarkable cheaper and highly available alternative to supplement culture mediums. On the other hand, previous reports showed that MK-801, an antagonist of GA receptor (NMDA receptor), decreased proliferation in primary human keratinocytes (2). Moreover, MK-801 topical application prevented hyperplasia induced by acetone (3), suggesting an anti-proliferative effect.

Glutamic acid has potent neurotoxic effects. Elevated amounts of GA lead to neuronal death in a process described as excitotoxicity (13-15). GA transporters are a potent GA uptake system, acting as a neuronal compensatory response for excitotoxicity. GA transporter showed to prevent disproportionate activation of GA receptors by constantly removing GA from the extracellular space (16-18). For the first time, we identified the excitotoxic GA concentration. In vivo, $100 \mathrm{mM}$ GA decreased keratinocyte viability and topical GA decreased $\mathrm{Bc} / 2$ and $\mathrm{Bax}$ expression. Altogether, our results support the excitotoxicity effect of higher concentration of pH-neutralized GA in keratinocytes.

To understand the exogenous GA effect on the skin, we identified the Glutamic acid transporter landscape at single-cell resolution in human and mice skins (Fig. 6d-e). 
Additionally, we showed SIc1a3 expression using quantitative PCR, and similar Slc1a3 (Glast) expression after exogenous GA application (Fig. 2c). Future research could help to identify the role of glutamic acid induced excitotoxicity and GA uptake system in the skin.

In terms of receptors, different subpopulation of glutamatergic cells has been extensively described (19-22). In the skin, previous reports identified the localization of the GA receptors and transporters in epidermis from rats and mice, as well as in human keratinocyte $(2,3,5)$. These studies showed similar cross-species characteristics: a smaller subpopulation of cells expressing receptors and transporters (2). Consistently with our findings, here we showed a small subpopulation of epidermal cells expressing GA receptors along skin with varying of intensity (Fig. 5a-b). Our results suggest that these glutamatergic keratinocytes are responsive to exogenous GA stimulation.

Previous reports showed that vascularization increases during anagen of the hair cycle and decreases during catagen and telogen phases. This angiogenesis process was spatially correlated with upregulation of VEGF (23). Also, the hypoxia-inducible factor (HIF) has shown to coordinate up-regulation of multiple genes controlling neovascularization, as Vegf (24). Here, we showed that after 14 days of GA topical treatment on the back skin of mice, Hif1a and Vegf expression increased with remarkable change in angiogenesis as previously shown (7).

A recent study supports that glutamic acid-mediated signaling could be involved in hair growth (25). The authors showed that glutamine, a similar molecule to glutamic acid, controls stem cell fate in the hair follicle. The capacity of the outer root sheath cells to return to the stem cell state requires suppression of a metabolic switch from glutamine metabolism and it is regulated by the mTORC2-Akt signaling axis (25). Similarly, our result showed that glutamic acid increases AKT phosphorylation and outer root sheath cells. In this way, our results further suggest that GA activates the hair cycle by stimulating the stem cells to differentiate into outer root sheath.

Altogether, the clinical relevance of our study is to open a possible complementary signaling mechanism for hair growth disorders, or even for aesthetic hair stimulation using topical GA.

We encourage further research to uncover the relationship between skin disorders and Glutamic acid. Also, our results could be the starting point to elucidate how Glutamic Acid from food intake or even from neuropsychiatric disorders could be associated with skin diseases. 


\section{Conclusion}

Glutamic acid receptors and transporters are present in the cell of the skin. Human keratinocytes cultured with GA can increase viability and proliferation. Also, topical GA decreases apoptotic related genes, accelerates hair growth in healthy mice, and increases vascularization. 


\section{Materials \& Methods}

\section{Experimental Animals}

8-week-old male Swiss mice $(n=6)$ were obtained from Breeding Animal Center of University of Campinas. Animals were maintained under pathogen-free conditions in individual cages on 1212 hours dark-light cycle, at $21-23 C^{\circ}$. Mice received chow and water ad libitum. Mice were anesthetized with intraperitoneal injections (according to the body weight), using ketamine hydrochloride $80 \mathrm{mg} / \mathrm{kg}$ and xylazine chlorhydrate $8 \mathrm{mg} / \mathrm{kg}$. Under general anesthesia animal hair was removed using mechanical razor and depilatory cream (Veet) on the back of each animal $(1 \mathrm{~cm} \times 2.5 \mathrm{~cm})$. The back of all animals was carefully cleaned to remove any trace of Veet cream. Animal experiments were approved by The Animal Ethical Committee at the University of Campinas, Brazil (certificate of approval no. 4930-1/2018).

\section{Topical Glutamic acid treatment}

The back of the mice was treated once daily using different concentrations of Glutamic acid. To uniform $200 \mu \mathrm{L}$ of treatment, we used different syringes preloaded with Vaseline (control), $0.1 \%, 0.5 \%, 1 \%$ or $10 \%$ glutamic acid (supplementary $1 \mathrm{c}$ ). We spread the treatment manually using gloves which were changing between one group and another. To avoid removal of treatment, mice used Elizabethan collars ( 8 of 14 days of treatment).

\section{Topical glutamic acid composition}

We made five different formulations from $0 \%$ to $10 \%$ of GA. Table 1 shows the different composition of each treatment. The $\mathrm{pH}$ of the formulations was adjusted using aqueous $\mathrm{NaOH}$ until the desired $\mathrm{pH} 5.5$ was achieved. This $\mathrm{pH}$ value was chosen to resemble the skin surface pH (Fig. 2b)

\section{Cell culture MTT and BrdU}

Human keratinocyte lineage (HaCat) passage $27-30$ were culture in $37 \mathrm{C}^{\circ}, 7 \% \mathrm{CO} 2$ incubator with DMEM medium supplemented with $5 \%$ FBS to $100 \%$ confluence in 6 -well plates. We replaced culture medium 2-3 times a week. 3-(4,5-dimethylthiazol-2-yl)-2,5diphenyltetrazolium bromide ( $\mathrm{MTT}$ ) assay was used to analyze cell viability as previously described. MTT solution was prepared in Krebs-HEPES buffer (10 mM HEPES, $1.2 \mathrm{mM} \mathrm{MgCl}$, $144 \mathrm{mM} \mathrm{NaCl}, 11 \mathrm{mM}$ glucose, $2 \mathrm{mM} \mathrm{CaCl} 2$, and $5.9 \mathrm{mM} \mathrm{KCl}$ ). After $100 \%$ confluence (6-well plates), HaCat were incubated with the different concentration of Glutamic acid in DMEM without FBS for 1, 2 and 4 days. After treatment, the medium was removed and MTT solution 
$(0.5 \mathrm{mg} / \mathrm{mL})$ was added to each well and incubated at $37{ }^{\circ} \mathrm{C}$ for $3 \mathrm{hrs}$. Then the solution was removed and $300 \mu \mathrm{L}$ of DMSO was added and incubated in dark at $60 \mathrm{rpm}$ shaker. The absorbance product was measured at a wavelength of $540 \mathrm{~nm}$ in a microplate reader (Globomax). All cell culture experiments were in quadruplicates. BrdU experiments were performed as previously described (26). Briefly, to assess the effect of GA on cell proliferation, HaCat human keratinocytes were maintained in Dulbecco's modified Eagle's medium (DMEM, Gibco) containing $4.5 \mathrm{~g} / \mathrm{L}$ glucose, $4 \mathrm{mM}$ L-glutamine, $100 \mathrm{units} / \mathrm{mL}$ of penicillin, $100 \mu \mathrm{g} / \mathrm{mL}$ of streptomycin, and $10 \%$ fetal bovine serum (FBS). Incubation conditions were $37{ }^{\circ} \mathrm{C}$ in $5 \%$ $\mathrm{CO} 2 /$ humidified air. HaCat cells were plated on coverslips in 24 -well plates ( $1 \times 10^{5}$ cells/well) and exposed to GA for $48 \mathrm{~h}$ (10 and $100 \mathrm{mM}$ ) in DMEM without FBS. After treatments, cells were incubated with BrdU (10 $\mu \mathrm{M}$, Sigma) for $3 \mathrm{~h}$, then fixed with 4\% PFA in $0.1 \mathrm{M}$ PBS for 10 min at RT. For BrdU staining, cells were washed with PBS, and DNA was denatured with $1 \mathrm{~N} \mathrm{HCl}$ for $1 \mathrm{~h}$ at RT. Cells were blocked for $1 \mathrm{~h}$ in blocking solution containing $10 \%$ goat serum and $0.2 \%$ Triton $\mathrm{X}-100$ in PBS, followed by an incubation with a primary (rat anti-BrdU; 1:200; Ab6326); and secondary goat anti-rat FITC (1:200; sc2011) antibodies prepared in 3\% goat serum/ $0.2 \%$ Triton X-100 in PBS, and incubated overnight and $2 \mathrm{~h}$, respectively. The nuclei were labeled with DAPI and coverslips were mounted onto glass slides for microscope imaging. Images were captured on fluorescence microscopy (Olympus BX41). The results of BrdU immunopositivity cells represent the average of 3 coverslips per experimental replicate, where 3 fields were imaged per coverslip and averaged. The number of immunopositive cells was quantified per image using the ImageJ software and are expressed as percentage relative to total DAPI nuclei.

\section{Animal photo Documentation}

Hair growth processes were photo documented using a D610 Nikon digital camera (Nikon Systems, Inc., Tokyo, Japan). To secure a similar distance from camera to treated skin site, we used a stand and the same person to take photos.

\section{Histology}

After 14 days of treatment, tissues were harvested and fixed by immersion in formaldehyde overnight. Any traces of formaldehyde were removed by 3 washes of PBS 1x. The tissue were processed in alcohol at different concentrations $(70 \%, 80 \%, 95 \%$, and $100 \%)$, xylol, and paraffin, before being fixed in paraffin blocks, and sectioned at $5.0 \mu \mathrm{m}$. 3 to 5 sessions were placed on microscope slides pretreated with poly-L-lysine. To evaluate cell and extracellular 
matrix morphology, the skin sections were stained with hematoxylin and eosin (H\&E). The sections were incubated with hematoxylin for $30 \mathrm{~s}$, rinsed in water, incubated for $30 \mathrm{~s}$ with eosin, rinsed again in water, and dehydrated. The slides were mounted in Entellan ${ }^{\oplus}$ and then analyzed; digital images were captured under bright-field microscopy.

Protein-Protein Interaction Networks. Protein functional interaction networks were performed using STRING v11. The default functional interaction network was configured to evidence meaning of network edges, experiments, and databases in active interaction sources. Mus musculus organism was visualized by known molecular action. We analyzed two Biological Process using Gene Ontology Term from Mouse Genome Informatics database. A permalink webpage of Glutamate receptor (GO:0007215) and hair follicle (GO:0042633) Gene ontologies Interaction network is accessible through https://version-11-0.stringdb.org/cgi/network.pl?taskId=IKUAbEZgGkVu for selected genes and https://version-110.string-db.org/cgi/network.pl?networkld=7i1fMIP01xqT for all genes.

Single-cell RNA sequencing data acquisition, filtering, and processing. In silico analyses were performed using a HP ENVY 17 Leap Motion SE NB PC notebook with 16GB RAM and fourcores Intel i7 processor. Sample expression matrices (mice and humans) were downloaded from Gene Expression Omnibus and European Genome-phenome Archive: GSE67602 and EGAS00001002927. Cells were filtered by their total number of reads, by their number of detected genes and by their mitochondrial percentage. For mice we used nFeature_RNA $>10$ and $<6000$, nCount_RNA $>100$ and $<50000$, percent.mt $<9.5$ settings. For humans we used nFeature_RNA > 100 and < 5000, nCount_RNA > 100 and $<25000$ \& percent.mt < 6 settings. Samples were processed in Seurat v3.1.5 using the default Seurat workflow. For clustering and visualization, we used the default Seurat pipeline gold standard and dot plot visualization. Cluster names were annotated to cell types accordingly original articles of Cheng et al. and Joost et al.

\section{Western Blotting}

For the immunoblot experiments, the tissues were homogenized in RIPA lysis buffer (150 mM $\mathrm{NaCl}, 50 \mathrm{mM}$ Tris, $5 \mathrm{mM}$ EDTA, 1\% Triton $\mathrm{X}-100,0.5 \%$ sodium deoxycholate, $0,1 \%$ sodium dodecyl sulfate, and supplemented with protease inhibitors). Insoluble material was removed by centrifugation $(11,000 \mathrm{rpm})$ for $40 \mathrm{~min}$ at $4{ }^{\circ} \mathrm{C}$, and the supernatant was used for protein quantification by the biuret reagent protein assay. Laemmli buffer (0.5 M Tris, 30\% glycerol, $10 \%$ SDS, 0.6 M DTT, 0.012 bromophenol blue) was added to the samples. One hundred 
micrograms of proteins were separated by SDS-PAGE and transferred to nitrocellulose membranes (Bio-Rad) using a Trans-Blot SD Semi-Dry Transfer Cell (Bio-Rad) for 1 hour at $17 \mathrm{~V}$ (constant) in buffer containing methanol and SDS. Blots were blocked in a $5 \%$ skimmed milk powder solution in TBST ( $1 \times$ TBS and $0.1 \%$ Tween 20 ) for $2 \mathrm{~h}$ at RT, washed with TBST, and incubated with the primary antibodies for $24 \mathrm{~h}$ at $4{ }^{\circ} \mathrm{C}$. The primary antibodies used were antipCaMKII (Abcam, ab32678) and anti-Fyn3 (Santa Cruz, sc-16). HRP-coupled secondary antibodies (1:5000, Thermo Scientific) were used for detection of the conjugate by chemiluminescence and visualization by exposure to an Image Quant LAS4000 (GE Healthcare, Life Sciences). Anti- $\beta$-actin (Abcam, ab8227) was used as a loading control. The intensities of the bands were digitally determined by densitometry, using Image J software (National Institutes of Health).

\section{Immunohistochemistry}

Skin expressions of Grin1, Grin2a, Grin2b, and Grin2c were identified by immunohistochemical staining. Immunohistochemistry was performed using the skin samples $(n=5)$. Tissue samples were immersed in $4 \%$ formaldehyde overnight. Tissue samples were washed three times with PBS 1x, cryopreserved in sucrose $20 \%$ for 3 days and $40 \%$ for 1 week. Samples were then embedded in OCT and sectioned using a cryostat (Leica CM1860). The sections ( $20 \mu \mathrm{m}$ ) were immunostained with the following primary antibodies: Grin1 (1:100, sc1467), Grin2a (1:100, sc1468), Grin2b (1:100, sc1469), Grin2c (1:100, sc9057), BrdU (1:200, ab6326) and keratin 14 (1:100, sc53253). VECTASHIELD with DAPI was used as a mounting medium for nuclear visualization. Images were obtained using a confocal microscope (Leica TCS SP5 II). For the in vivo $\mathrm{BrdU}$ experiment, we treated the mice intraperitoneally as previously described. Brightly, we applied one single injection of $\mathrm{BrdU}(150 \mathrm{mg} / \mathrm{kg}$ in buffer citrate) $3 \mathrm{~h}$ before skin harvest.

\section{Real-time Quantitative Polymerase Chain Reaction (RT-qPCR)}

The total RNA content was extracted from the tissue using TRlzol reagent (Invitrogen). For each sample, two micrograms of RNA were reverse transcribed to CDNA, according to the manufacturer's instructions (High-Capacity cDNA Reverse Transcription Kit, Life Technologies). Gene expression analysis via RT-qPCR was performed using TaqMan Universal PCR Master Mix (7500 detection system, Applied Biosystems). Primers used were: Bcl2: Mm00477631_m1; Bax: Mm00432051_m1; Casp9: Mm00516563_m1; F4/80: Mm00802529_m1; Mcp1: Mm00441242_m1; Il1b: Mm00434228_m1; TNFa: Mm00443258_m1; Il10: Mm01288386_m1; Grin1: Mm00433790_m1; Glast: Mm00600697_m1; Hif1a: Mm00468869_m1; Vegf: 
Mm00437306_m1; and Cd31: Mm01242576_m1 (Thermofisher). Analyses were run using $4 \mu \mathrm{L}$ (10 ng/ $\mu \mathrm{L}$ ) cDNA, $0.625 \mu \mathrm{L}$ primer/probe solution, $1.625 \mu \mathrm{L} \mathrm{H2O}$, and $6.25 \mu \mathrm{L} 2 \mathrm{X}$ TaqMan Universal MasterMix. GAPDH (Mm99999915_g1) was employed as a reference gene. Gene expression was obtained using the SDS System 7500 software (Applied Biosystems).

\section{Conflict of Interest}

The authors declare no competing financial interests.

\section{Acknowledgements}

The authors are grateful to Marcio Alves da Cruz, Joseane Morari, Vanessa Bobbo, Erika Anne Roman and Gerson Ferraz for technical assistance. This study was supported in part by the Coordenação de Aperfeiçoamento de Pessoal de Nível Superior - Brasil (CAPES) - Finance Code 88882.434714/2019-01. 


\section{Figures}

Figure 1. Effects of GA treatment in vitro and in vivo.

Experimental design of cell culture experiment (a). HaCat cells viability results after 1 day (b) and 2 days (c) of exposure. Immunostaining of BrdU/Dapi positive cells (d). HaCat cells were exposed to DMEM (control group), GA 10 or $100 \mathrm{mM}$ for $48 \mathrm{~h}$ and finally $3 \mathrm{~h}$ with BrdU, scale bar $50 \mu \mathrm{m}$. Proportion of BrdU-immunoreactive increased after exposure to $\mathrm{GA} 10 \mathrm{mM}(\mathrm{g})$. Data is presented as mean \pm SEM. $N=4$ per group. $p=0.03$ t-test Control vs Glutamic Acid $10 \mathrm{mM} ; \mathrm{p}=0.03$ One-way ANOVA. Experimental design of cell culture experiment (e). Different GA concentrations were equal to $5.5 \mathrm{pH}$ (f). Bcl2, Bax, Casp9, F4/80, Mcp1, II1 , Tnf $\alpha$, II10, Grin1, and Glast gene expression on skin samples after 14 days of GA treatment $(\mathrm{h}) *<\mathrm{p} 0.05$ ANOVA.

\section{Figure 2. GA stimulates hair growth.}

Dose-response of topical GA application on the back of mice with Vaseline (CTL) and $0.1 \%$, $0.5 \%, 1 \%$ and $10 \%$ GA for 14 days (a-c). Hair growth effect (a), H\&E staining (outer root pointed with yellow arrows), and upside-down back skin samples showing vessel differences (c). Quantification of blood vessels area after 14 days of GA skin treatment (d). Gene expression of Hif1a, Vegf and Cd31 from back skin after 14 days of GA treated (e).

\section{Figure 3. Topical GA increased BrdU+ cells.}

BrdU+ cells after 14 days of GA treatment (a). Yellow arrows indicate BrdU+ cells. Top scale bar $250 \mu \mathrm{m}$ and bottom $100 \mu \mathrm{m}$ (a). Characterization of different subunits Grin2b, Grin1, Grin2a, and Grin2c of GA receptor NMDA expressed in the skin of untreated mice $(b-c)$. Yellow arrows indicate colocalization of $\mathrm{K} 14+\mathrm{Grin} 2 \mathrm{~b}+$ cells (b). Positive BrdU epidermal and hair follicle cells (d). 


\section{Figure 3. Cross-species skin GA receptor landscape using single-cell RNA sequencing}

Generation Glutamic acid landscape using public data reveals GA distribution at single cell resolution in mice and humans. Schematic representation of our single-cell RNA sequencing analysis (a). Human Epidermal Glutamate receptors (b) and transporter expression (c). Mice Epidermal Glutamate receptors (d) and transporter expression (e). Glutamic acid and hair cycle Protein-Protein Interaction Network. Glutamic acid and hair cycle interactome were retrieved with the data-mining toolkit string. Interactors of Glutamic acid and hair cycle ontologies were set as colored nodes. Yellow arrows indicated shared shell interactors (f). Protein quantification to confirm Protein-Protein Interaction Network prediction (g-i). Phosphorylation of AKT (g), phospho-CaMKII (h) and Fyn quantification (i). 


\section{Bibliography}

1. Zhou Y, Danbolt NC. Glutamate as a neurotransmitter in the healthy brain. Journal of Neural Transmission. 2014;121(8):799-817.

2. Genever PG, Maxfield SJ, Kennovin GD, Maltman J, Bowgen CJ, Raxworthy MJ, et al. Evidence for a novel glutamate-mediated signaling pathway in keratinocytes. J Invest Dermatol. 1999;112(3):337-42.

3. Fuziwara S, Inoue K, Denda M. NMDA-type glutamate receptor is associated with cutaneous barrier homeostasis. J Invest Dermatol. 2003;120(6):1023-9.

4. Woo S-H, Baba Y, Franco AM, Lumpkin EA, Owens DM. Excitatory glutamate is essential for development and maintenance of the piloneural mechanoreceptor. Development. 2012;139(4):740.

5. Morhenn VB, Waleh NS, Mansbridge JN, Unson D, Zolotorev A, Cline P, et al. Evidence for an NMDA receptor subunit in human keratinocytes and rat cardiocytes. Eur J Pharmacol. 1994;268(3):409-14.

6. Nordlind K, Johansson O, Lidén S, Hökfelt T. Glutamate- and aspartate-like immunoreactivities in human normal and inflamed skin. Virchows Archiv B. 1993;64(1):75-82.

7. Thangavel P, Ramachandran B, Chakraborty S, Kannan R, Lonchin S, Muthuvijayan V. Accelerated Healing of Diabetic Wounds Treated with L-Glutamic acid Loaded Hydrogels Through Enhanced Collagen Deposition and Angiogenesis: An In Vivo Study. Scientific Reports. 2017;7(1):10701.

8. Semenza GL. Hypoxia-inducible factor 1: master regulator of $\mathrm{O} 2$ homeostasis. Curr Opin Genet Dev. 1998;8(5):588-94.

9. Chen L, Endler A, Shibasaki F. Hypoxia and angiogenesis: regulation of hypoxiainducible factors via novel binding factors. Experimental \& Molecular Medicine. 2009;41(12):849-57.

10. Ferrara N. VEGF and the quest for tumour angiogenesis factors. Nature Reviews Cancer. 2002;2(10):795-803.

11. Rumney RMH, Lanham SA, Kanczler JM, Kao AP, Thiagarajan L, Dixon JE, et al. In vivo delivery of VEGF RNA and protein to increase osteogenesis and intraosseous angiogenesis. Scientific Reports. 2019;9(1):17745.

12. Leung DW, Cachianes G, Kuang WJ, Goeddel DV, Ferrara N. Vascular endothelial growth factor is a secreted angiogenic mitogen. Science. 1989;246(4935):1306-9.

13. Dong $X-x$, Wang $Y$, Qin Z-h. Molecular mechanisms of excitotoxicity and their relevance to pathogenesis of neurodegenerative diseases. Acta Pharmacologica Sinica. 2009;30(4):37987.

14. Piña-Crespo JC, Sanz-Blasco S, Lipton SA. Concept of Excitotoxicity via Glutamate Receptors. In: Kostrzewa RM, editor. Handbook of Neurotoxicity. New York, NY: Springer New York; 2014. p. 1015-38.

15. Crabbé M, Dirkx N, Casteels C, Laere KV. Excitotoxic neurodegeneration is associated with a focal decrease in metabotropic glutamate receptor type 5 availability: an in vivo PET imaging study. Scientific Reports. 2019;9(1):12916.

16. O'Donovan SM, Sullivan CR, McCullumsmith RE. The role of glutamate transporters in the pathophysiology of neuropsychiatric disorders. npj Schizophrenia. 2017;3(1):32.

17. Parsons MP, Vanni MP, Woodard CL, Kang R, Murphy TH, Raymond LA. Real-time imaging of glutamate clearance reveals normal striatal uptake in Huntington disease mouse models. Nature Communications. 2016;7(1):11251. 
18. Paoletti $\mathrm{P}$, Bellone $\mathrm{C}$, Zhou Q. NMDA receptor subunit diversity: impact on receptor properties, synaptic plasticity and disease. Nature Reviews Neuroscience. 2013;14(6):383-400.

19. Tatti R, Bhaukaurally K, Gschwend O, Seal RP, Edwards RH, Rodriguez I, et al. A population of glomerular glutamatergic neurons controls sensory information transfer in the mouse olfactory bulb. Nature Communications. 2014;5(1):3791.

20. Heikkinen AE, Möykkynen TP, Korpi ER. Long-lasting Modulation of Glutamatergic Transmission in VTA Dopamine Neurons after a Single Dose of Benzodiazepine Agonists. Neuropsychopharmacology. 2009;34(2):290-8.

21. Montardy Q, Zhou Z, Lei Z, Liu X, Zeng P, Chen C, et al. Characterization of glutamatergic VTA neural population responses to aversive and rewarding conditioning in freely-moving mice. Science Bulletin. 2019;64(16):1167-78.

22. Yamaguchi T, Qi J, Wang H-L, Zhang S, Morales M. Glutamatergic and dopaminergic neurons in the mouse ventral tegmental area. European Journal of Neuroscience. 2015;41(6):760-72.

23. Yano K, Brown LF, Detmar M. Control of hair growth and follicle size by VEGF-mediated angiogenesis. The Journal of clinical investigation. 2001;107(4):409-17.

24. Oladipupo S, Hu S, Kovalski J, Yao J, Santeford A, Sohn RE, et al. VEGF is essential for hypoxia-inducible factor-mediated neovascularization but dispensable for endothelial sprouting. Proceedings of the National Academy of Sciences of the United States of America. 2011;108(32):13264-9.

25. Kim CS, Ding X, Allmeroth K, Biggs LC, Kolenc Ol, L'Hoest N, et al. Glutamine Metabolism Controls Stem Cell Fate Reversibility and Long-Term Maintenance in the Hair Follicle. Cell Metabolism. 2020.

26. Engel DF, Grzyb AN, de Oliveira J, Pötzsch A, Walker TL, Brocardo PS, et al. Impaired adult hippocampal neurogenesis in a mouse model of familial hypercholesterolemia: A role for the LDL receptor and cholesterol metabolism in adult neural precursor cells. Molecular Metabolism. 2019;30:1-15. 
bioRxiv preprint doi: https://doi.org/10.1101/2020.09.27.315523; this version posted September 28, 2020. The copyright holder for this preprint (which was not certified by peer review) is the author/funder. All rights reserved. No reuse allowed without permission. 
L Grank add

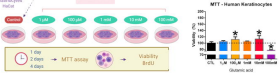

(1) 1-day exposure
2 days exposure

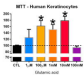

ANDVA $P=005$

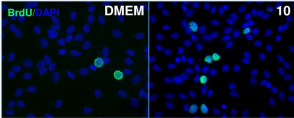

\section{0}

100

Dasy 0

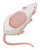

Trached once daly

ense

Tapical

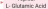

$\dot{m \infty \infty} \infty$

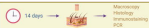

h
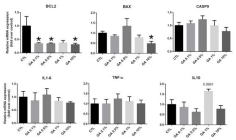

GA
$=01 \%$
$=05 \%$
$=18$

$=18$
$-10 \%$
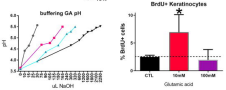

ARXOVA + $0<005$

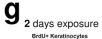

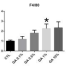

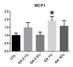

(ast

Guar

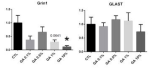




\section{Glutamic acid}

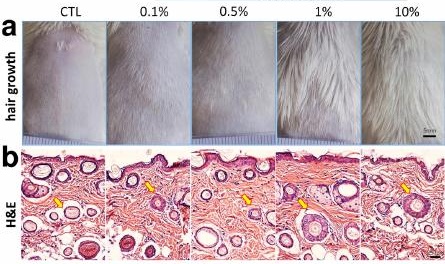

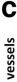

d

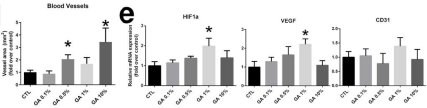




\section{a}

b Human Epidermal Glutamate receptor
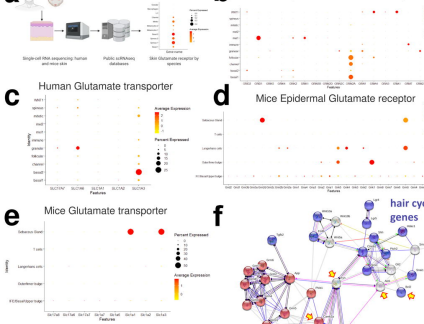

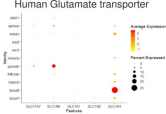

C
Mice Epidermal Glutamate receptor

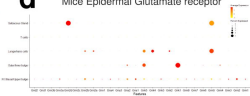

g puct?

CTL $\frac{\mathrm{GA}}{1 \% 10 \% \mathrm{CTL}} \frac{\mathrm{GQ}}{1 \% 1 \mathrm{D} \%}$ AKT2 2

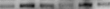

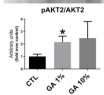

glutamate receptor signaling pathway genes
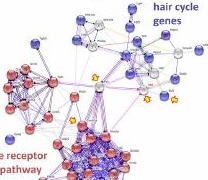